\title{
Implicit Motor Learning and Complex Decision Making in Time-Constrained Environments
}

\author{
R. S. W. Masters \\ J. M. Poolton \\ J. P. Maxwell \\ Institute of Human Performance \\ The University of Hong Kong, China
}

\author{
M. Raab \\ Institute for Movement Sciences and Sport \\ University of Flensburg, Germany
}

\begin{abstract}
The cost-effectiveness of the implicit (procedural) knowledge that supports motor expertise enables surprisingly efficient performance when a decision and an action must occur in close temporal proximity. The authors argue that if novices learn the motor component of performance implicitly rather than explicitly, then they will also be efficient when they make a decision and execute an action in close temporal proximity. Participants $(N=$ $35)$ learned a table tennis shot implicitly or explicitly. The authors assessed participants' motor performance and movement kinematics under conditions that required a concurrent low-complexity decision or a concurrent high-complexity decision about where to direct each shot. Performance was disrupted only for participants who learned explicitly when they made high-complexity decisions but not when they made low-complexity decisions. The authors conclude that implicit motor learning encourages cognitively efficient motor control more than does explicit motor learning, which allows performance to remain stable when time constraints call for a complex decision in tandem with a motor action.
\end{abstract}

Keywords: analogy learning, cognitive load, expertise, explicit instructions, movement kinematics, procedural knowledge

A ppropriate decision making requires the integration of perceptual information with knowledge obtained from previous experiences and places varying demands on cognitive resources, depending on the complexities of the task (e.g., Raab, 2003; Sève, Saury, Theureau, \& Durand, 2002) and the extent to which performance depends on working memory (Jameson, Hinson, \& Whitney, 2004).

Expert performers' ability to process the multiple streams of information that they need for effective perceptionaction interaction characterizes the highly efficient way in which they interface with their specialist environment. If the environment is time constrained, then performers must make decisions and execute movements in close temporal proximity. In tennis, for example, hitting a forehand win- ner past an opponent while running demands appropriate movement selection (e.g., should the shot be hit down the line, across the court, deep, short, with underspin, or with topspin?) coupled with immediate and effective movement execution.

One explanation for experts' highly efficient decisionmaking skills is that the nature of the knowledge structures that support their motor performance gradually changes over time, with an increasing degree of implicit (unconscious) control and a decreasing level of explicit (conscious) control. In contrast to conscious control processes, implicit processes are faster and are organized as sophisticated procedural knowledge that can be applied without conscious thought (e.g., Anderson, 1983; Lewicki, Hill, \& Czyewska, 1992; Masters \& Maxwell, 2004; Shiffrin \& Schneider, 1977; Willingham, 1998). Implicit processes are therefore independent of working memory, which leaves the expert with sufficient resources to perform other tasks, such as decision making (for a review of the theoretical architecture and function of working memory, see Baddeley, 2003). In contrast, explicit processes depend on working memory for the retrieval of consciously accessible (declarative) knowledge so that the motor system can control movement online (Maxwell, Masters, \& Eves, 2003). Because highly explicit motor behavior depends on working memory, the demands that result from multiple task requirements are likely to overload the performer and disrupt performance.

In a test of that theory, Poolton, Masters, and Maxwell (2006) argued that disrupted motor performance is less likely to occur if the motor component of performance

Correspondence address: R. S. W. Masters, Institute of Human Performance, The University of Hong Kong, 111-113 Pokfulam Road, Hong Kong SAR, China. E-mail address: mastersr@hku.hk 
is learned implicitly rather than explicitly, because more resources will be available for decision making. Investigators believe that implicit motor learning techniques advance implicit control and have found that those techniques engender resistance to disruption from additional cognitive loads (e.g., Maxwell, Masters, Kerr, \& Weedon, 2001), moderate psychological pressure (Hardy, Mullen, \& Jones, 1996; Masters, 1992), and physiological exertion (Poolton, Masters, \& Maxwell, 2007).

Using a table tennis task, Poolton et al. (2006) trained participants explicitly (by using step-by-step instructions) and implicitly (by providing an analogical instruction; see Liao \& Masters, 2001) and tested their performance in conditions that required that they make a low-complexity decision or a high-complexity decision regarding the direction in which they hit the ball. Differences between the treatment conditions were evident only when participants had to make high-complexity decisions, and their motor performance was disrupted in the explicit condition but not in the implicit condition. In fact, performance in the implicit condition appeared to improve when participants had to process high-complexity decisions, which suggests that processing efficiency is greater in implicitly trained participants than in explicitly trained participants. The latter group appeared to be unable to switch efficiently between the tasks or to process the tasks in parallel without disruption to motor output (Hazeltine, Ruthruff, \& Remington, 2006).

Firm conclusions about the underlying reasons for the findings of Poolton et al. (2006) are clouded by the possibility that motor adaptations were ongoing during the lowcomplexity and high-complexity decision-making tests. Participants had to direct shots to a central target during the learning phase but to the left or the right side of the table during the decision-making phase. Participants' differential adaptation to the new task demands in the two conditions may have occurred during the decision-making phase. Implicit (analogy) learners' better adaptation would have hidden the disruptive effect of the high-complexity decision.

In the present experiment, we addressed that possible confound by interspersing the decision-making tests between two transfer tests in which we required participants to hit balls alternately to the left and right of center. The transfer tests served as a measure of baseline transfer performance and allowed us to assess the amount of adaptation in the two conditions during the test phase (superior performance in the second transfer test would be indicative of learning). We also performed kinematic analysis of movements to identify some of the movement characteristics associated with implicit (analogy) learning and explicit (step-by-step) learning and to examine the kinematic effect of producing a movement and a decision concurrently. We expected that participants in the implicit condition would show stable (or improved) motor performance and unperturbed movement kinematics when we required them to make concurrent lowor high-complexity decisions. In contrast, we expected that participants in the explicit condition would show reduced motor performance and perturbed movement kinematics as a result of those requirements (e.g., increased jerk; Maxwell et al., 2003). We also expected implicit (analogy) learners to report less explicit (declarative) knowledge of their movements than explicit learners would.

\section{Method}

\section{Participants}

We randomly assigned 35 undergraduate students (age $=$ $21.3 \pm 2.27$ years $[M \pm S D]$ ) from the University of Hong Kong to either an analogy $(n=17)$ or an explicit $(n=$ 18) condition. All participants were right-handed and had little or no table tennis experience. Participants provided informed consent and received $\$ 100 \mathrm{HK}$ (approximately $\$ 13$ US) for participation.

\section{Apparatus}

Participants performed the experiment on a standard table tennis table (Komann KBT-2018). At one end was a table tennis ball server (Newgy Robo-pong 2000) that discharged $40-\mathrm{mm}$ balls at a frequency of $30 \mathrm{balls} / \mathrm{min}$. The server directed balls down the center line of the table with backspin, and the balls ascended to approximately $20 \mathrm{~cm}$ at the table's edge. We placed 100 balls (50 white and 50 yellow) in the ball storage hopper and mixed them regularly to ensure that they were randomly dispersed. We adapted the ball server to prevent identification of the ball's color before it was discharged. All participants used a Donic Waldner 500 table tennis bat. A reflective marker attached to the distal edge of the bat was tracked by a six-camera Qualisys (Gothenburg, Sweden) motion capture system, which allowed us to analyze bat movement kinematics during task execution.

Below the server, we marked six large squares $(50 \mathrm{~cm} \times$ $50 \mathrm{~cm}$ ) on the table in two rows (see Figure 1). Each square in the row farthest from the participant housed a concentric target $(25 \mathrm{~cm} \times 25 \mathrm{~cm})$. During the learning trials, participants aimed to hit the central target. We awarded participants 3 points for hitting Zone 2 and 3 points for hitting Zone 5. A ball landing in any other zone received 1 point. In the test phase, participants used the targets on the left or right of the table. We awarded them 3 points for hitting Zones 1 or 3 and 3 points for hitting Zones 4 or 6 . We awarded 1 point for hitting any other zone. We gave balls hit to the incorrect side of the table or out of a marked zone a score of 0 . For example, we awarded 1 point for a ball directed (correctly) to the right-hand target that hit Zone 2, 5,8 , or 9 and 0 points for a ball directed to the right-hand target that hit Zone 1, 4, or 7 .

\section{Procedure}

We informed participants that the task was to develop an accurate topspin forehand shot. We told them that their objective in the task was to return shots, with topspin, toward Zone 2 (see Figure 1). We explained the ball rotation generated 


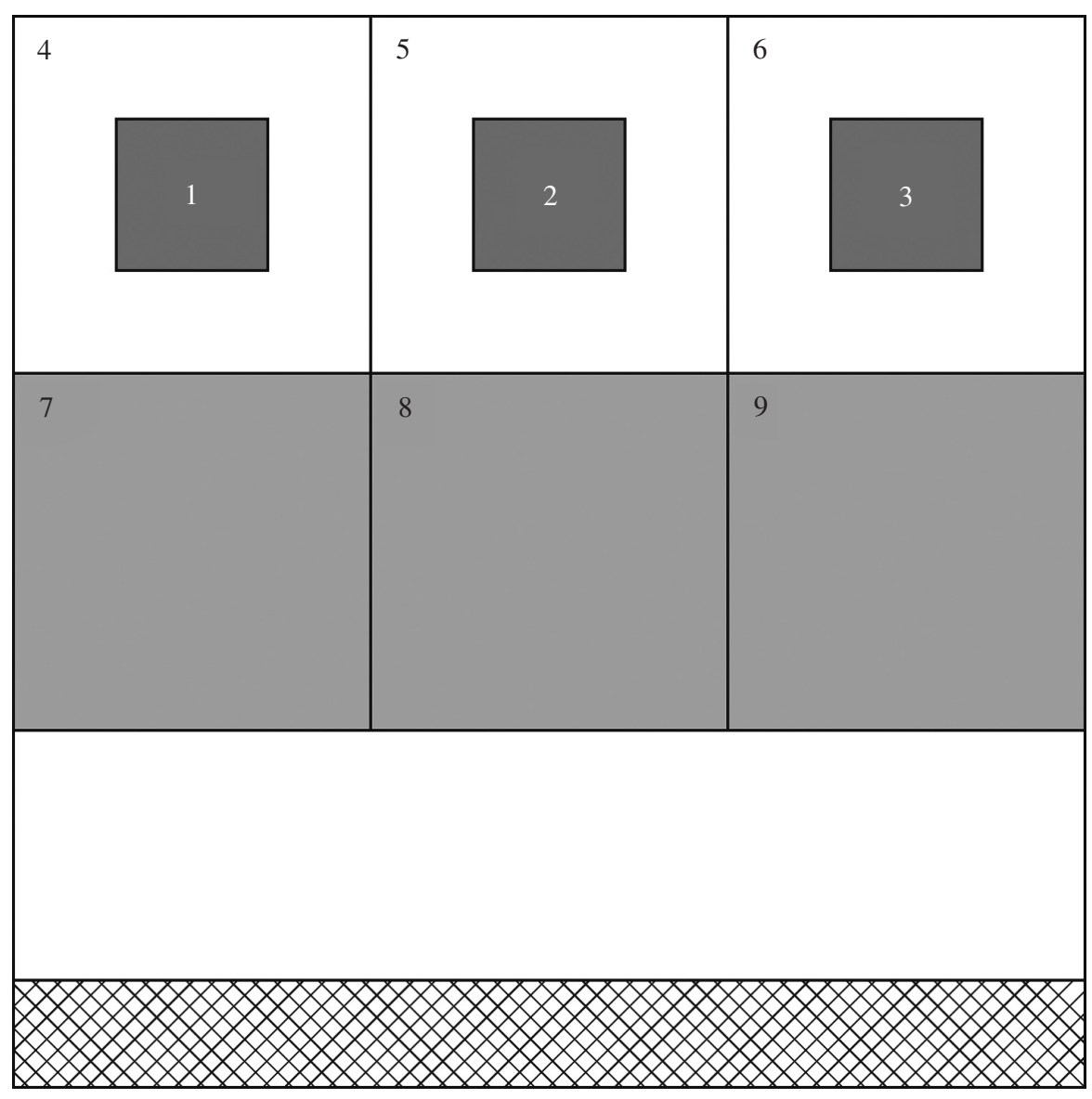

FIGURE 1. Target areas. Participants targeted Zone 2 during the learning phase, whereas they targeted Zones 1 and 3 during the test phase.

by a topspin forehand and asked participants to hold the bat with a Western shake-hands grip (Sneyd, 1994). We provided separate instructions in the two treatment conditions. We presented six step-by-step instructions in the explicit treatment condition (see Appendix), whereas in the analogy condition, we presented a single analogical instruction: "Move the bat as if it is traveling up the side of a mountain" (Poolton et al., 2006). At no point did we demonstrate a topspin forehand. Participants completed 300 trials in fifteen 20-trial blocks, over a 1-hr learning period. We emphasized the importance of following the instructions before each block of trials. If a participant failed to hit shots with topspin within a block of trials (as judged by the experimenter), then we again explained the appropriate ball rotation. We gave no feedback concerning the correctness of a participant's technique. After the learning phase, we administered a declarative knowledge protocol. In the protocol, we asked participants to report in as much detail as possible any movements, methods, or techniques they remembered using to perform the task.

In the test phase, we interspersed low- and high-complexity tests between two transfer tests. Each test consisted of two blocks of 20 trials (we later analyzed the two blocks togeth- er as one 40-trial block). In the two transfer tests, instead of hitting toward the central target, participants were to hit the balls to targets on the left (Zone 1) or the right (Zone 3) side of the table in an alternating sequence (i.e., the first ball to the right, the second to the left, the third to the right, and so on). In the low- and high-complexity tests, ball color specified the location of the target. In the low-complexity test, participants were to hit white balls to the right and yellow balls to the left. Before motor performance, we evaluated participants' ability to make correct decisions in a 20-trial block (decision-only test) in which participants verbally indicated whether the ball should be hit left or right.

In the high-complexity test, we alternated the ball color and target representation after every two balls. For Trials 1 and 2, as in the low-complexity test, participants had to hit white balls to the right and yellow balls to the left. In Trials 3 and 4, we switched the ball color and target representation so that participants had to hit white balls to the left and yellow balls to the right. Trials 5 and 6 reverted to white-right and yellow-left, Trials 7 and 8 reverted to white-left and yellow-right, and so on. As in the low-complexity test, we evaluated participants' abil- 
ity to make correct decisions by using a decision-only test. On occasions when a participant forgot the correct ball color sequence, he or she notified the experimenter, who then asked the participant to resume from the initial sequence (e.g., for the first two balls, hit white balls to the right-hand target).

We computed the total score in each block of 20 trials (maximum score $=60$ ) as a percentage and used that score as the dependent variable in the learning phase. In the test phase, we examined the manipulation of decision complexity by totaling the number of correct decisions made in the low- and the high-complexity tests when the decisions (a) were made without a motor response and (b) were accompanied by a motor response. To assess motor performance in the test phase and to avoid the possibility of confounding by a tradeoff of decision versus motor performance, we computed our dependent variable from the mean performance only on trials in which participants made a correct decision.

During the high-complexity test, we asked participants to immediately report occasions when they forgot the sequence of the task. It became clear, however, that participants had on occasion unwittingly forgotten the order that they had to follow. A string of correct responses would be followed by a series of incorrect responses, but the order of the incorrect decisions was not random. It seemed that participants inadvertently missed a ball in the sequence and continued the sequence from the next ball. As a result, the ball sequence that participants followed on those occasions was not matched to the experimental ball sequence. Thus, their performance scores did not always reflect task proficiency. To address that problem, we identified the ball in the sequence that the participant had missed, and we then rescored performance from that point on. Because of the subjective nature of that procedure, a second rater independently rescored the number of correct decisions made in the high-complexity test. Significant correlations between the two raters in both the decision-only test and the decision-plus-motor-performance test confirmed the accuracy of the primary rater's scoring; intraclass correlation coefficients $(I C C s)=.89$ and $.84, F_{\mathrm{S}}(1,34)=16.81$ and 11.14 , respectively, both $p \mathrm{~s}<.001$.

We used the motion capture system to track bat movement in the first and final blocks of learning and in the first 20 trials of each block in the test phase. The system operated at a sampling frequency of $60 \mathrm{~Hz}$ and tracked the reflective marker attached to the bat to within $1 \mathrm{~mm}$ of error. The $x$, $y$, and $z$ coordinates specified the relative position of the bat at each point in time. From the output data, we extracted the three-dimensional (3D) coordinates of the bat marker trace for each trial. We defined a trial as the initial backward preparatory movement through the forward movement to the highest vertical point of the follow-through. From the coordinates, we computed peak and mean movement speeds by calculating the distance the bat marker traveled in 3D space (in meters) at each sampled time point and then dividing that distance by unit time (i.e. 1/60 s). We calculated peak and root mean square acceleration (RMSacc) and peak and root mean square jerk (RMSjerk) as the second and third time derivatives of distance, respectively. We also calculated trialto-trial variability $(S D)$ for each of the six kinematic measures, which yielded 12 dependent variables. We excluded data from 7 participants (3 analogy condition participants, 4 explicit condition participants) from kinematic analysis of the learning phase because of either system failure or poor-quality marker traces that prevented a comprehensive analysis. Thus, we included data from 14 analogy condition participants and 15 explicit condition participants in the learning phase analysis. We excluded data from 4 participants ( 1 analogy condition participant, 3 explicit condition participants) from analysis of the test phase. Thus, we included data from 16 analogy condition participants and 15 explicit condition participants in the test phase analysis.

Two independent raters scored the declarative knowledge protocols. The raters assessed the amount of information related to the mechanics of movement (e.g., "I turned my shoulders as I struck the ball" or "I kept the bat as much as possible on a vertical plane"). The 92 ICC value, $F(1$, $34)=22.51, p<.001$, showed a high level of concordance between the two raters' scores. We therefore averaged the scores from the independent raters for analysis.

\section{Results}

\section{Performance: Learning Phase}

We assessed the accuracy of topspin forehand performance during learning with a $2 \times 15$ (Group $\times$ Block) analysis of variance (ANOVA) with repeated measures on block and with Greenhouse-Geisser's epsilon adjustment to degrees of freedom in all cases. A main effect was found for block, $F(8.01,264.26)=23.26, p<.001, \eta^{2}=.41$, but not for group, $F(1,33)=0.66, p=.42, \eta^{2}=.02$. No Group $\times$ Block interaction was evident, $F(8.01,264.26)=0.74, p=$ $.65, \eta^{2}=.02$. As illustrated in Figure 2, the treatment conditions appeared to have similar learning outcomes.

\section{Kinematics: Learning Phase}

We found no differential effect of instructional method in the analysis of performance during the learning phase. We therefore did not expect main effects of group in any of the kinematic parameters. Performance increased over blocks, however, so we expected to see evidence of change over blocks in the kinematic parameters. The same rationale caused us to expect no interactions between group and block.

Univariate ANOVAs that we computed for each dependent measure demonstrated significant effects of block for mean speed, $F(1,26)=12.22, p<.005, \eta^{2}=.32$; peak speed, $F(1,26)=20.46, p<.001, \eta^{2}=.44$; peak $R M S$ acc, $F(1,26)=5.36, p<.05, \eta^{2}=.17$; and trial-to-trial variability of mean $R M S$ acc, $F(1,26)=6.19, p<.05, \eta^{2}=.19$. Mean and peak speed and peak RMSacc increased over learning, whereas trial-to-trial variability decreased. That pattern suggests that participants made shots with greater 


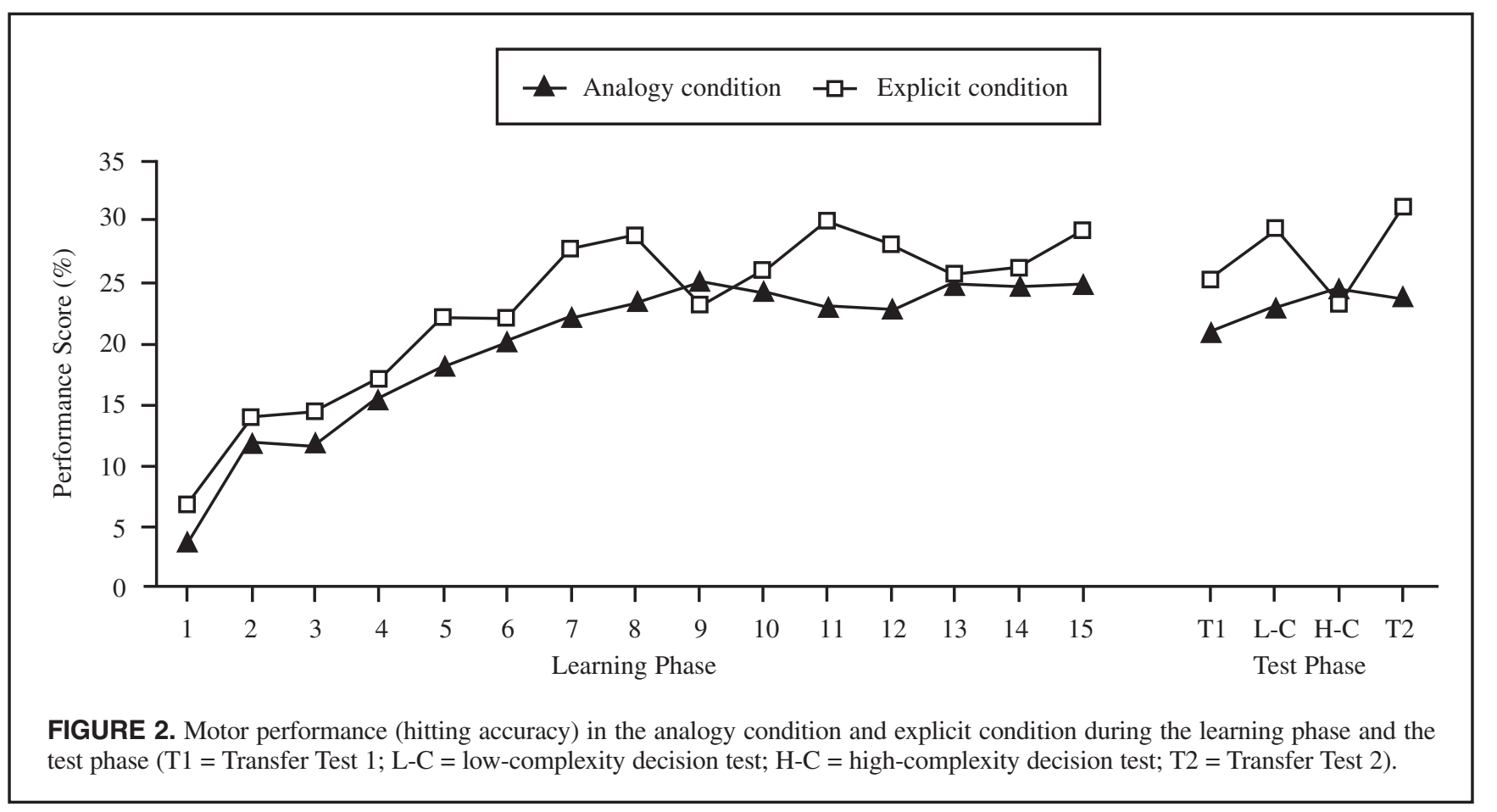

force and became more consistent with practice. Although changes were not significant for other variables, they followed the same pattern (i.e., increasing mean and peak values and decreasing trial-to-trial variability).

Contrary to expectations, group effects were evident for peak $R M S$ acc, $F(1,26)=12.18, p<.005, \eta^{2}=.32$; mean $R M S$ acc, $F(1,26)=9.77, p<.005, \eta^{2}=.27$; trial-to-trial variability of peak $R M S$ acc, $F(1,26)=4.44, p<.05, \eta^{2}=$ .15 ; peak $R M S$ jerk, $F(1,26)=14.80, p<.005, \eta^{2}=.36$; trial-to-trial variability of peak $R M S$ jerk, $F(1,26)=4.28$, $p<.05, \eta^{2}=.14$; mean RMSjerk, $F(1,26)=4.44, p<.05$, $\eta^{2}=.15$; and trial-to-trial variability of mean $R M S$ jerk, $F(1$, 26) $=5.94, p<.05, \eta^{2}=.19$. In all cases, larger values were evident for the analogy group. No interactions between group and block were found.

\section{Decision-Only Test}

A $2 \times 2$ (Group $\times$ Decision) ANOVA with repeated measures on number of correct verbal responses that participants made in the low- and high-complexity tests showed no main effect of group, $F(1,33)=1.22, p=.28, \eta^{2}=.04$, and no interaction, $F(1,33)=0.63, p=.43, \eta^{2}=.02$. A main effect of decision was evident, $F(1,33)=56.65, p<.001, \eta^{2}=$ .63. Participants made fewer correct decisions in the highcomplexity test $(M=81.71 \%)$ than in the low-complexity test $(M=98.86 \%)$, corroborating the effectiveness of the complexity manipulation.

\section{Motor Performance: Test Phase}

We assessed the ability of participants to adapt to hitting the ball to the left and right sides of the table rather than down its center by conducting a Group $\times$ Block (final
20 trials of learning vs. Transfer 1) ANOVA. The analysis showed a main effect of block only, $F(1,33)=9.84, p<$ $.005, \eta^{2}=.23$. Performance accuracy in the transfer test was lower, but the two conditions appeared to transfer to the new task demands in a similar manner.

We assessed motor performance in the test phase with a $2 \times 4$ (Group $\times$ Block) ANOVA with repeated measures; we used the percentage score per correct decision as the dependent variable. No significant effect of group was found, $F(1,33)=1.49, p=.23, \eta^{2}=.04$. However, a main effect of block, $F(2.81,92.64)=4.70, p<.01, \eta^{2}=.13$, and an interaction, $F(2.81,92.64)=4.30, p<.01, \eta^{2}=.12$, were evident. A posteriori analysis of simple main effects showed no effect of block for the analogy condition, $F(2.59,41.4)=$ $1.39, p=.26, \eta^{2}=.08$. However, the analysis revealed a significant effect for the explicit condition, $F(2.72,46.25)=$ $7.22, p<.005, \eta^{2}=.30$. As shown in Figure 2, participants in the explicit condition showed significantly poorer performance in the high-complexity test than in the lowcomplexity test, $p<.01$, and in the second transfer test, $p$ $<.005$. Moreover, participants in the explicit condition had superior motor performance in both the low-complexity test and Transfer 2 than in Transfer 1 , both $p s<.01$, which was indicative of continued learning during the test phase.

Assessment of the number of correct motor responses participants made in the low- and high-complexity tests yielded no main effect of group, $F(1,33)=2.08, p=.16, \eta^{2}$ $=.06$, or Group $\times$ Block interaction, $F(1,33)=.31, p=.58$, $\eta^{2}=.01$. However, an effect of block, $F(1,33)=71.21, p<$ $.001, \eta^{2}=.68$, was evident. Participants made more correct decisions in the low-complexity test $(M=94.72 \%)$ than in the high-complexity test $(M=78.14 \%)$. 


\section{Kinematics: Test Phase}

The performance results in the test phase allowed us to make specific predictions regarding changes in kinematic parameters of the movements in each treatment condition. The absence of performance change in the analogy condition suggests that an absence of changes in kinematic parameters should also be evident. Conversely, changes to key kinematic parameters should reflect the changes in performance in the explicit condition, particularly during the high-complexity decision test. To verify those predictions, we conducted separate univariate repeated measures analyses for each group, taking each of the 12 kinematic variables as a dependent measure.

We found no significant effects of block in the analogy condition; $p>.05$ in all cases. That result is consistent with participants' performance data (see Table 1). However, significant changes were evident in several of the kinematic parameters that we assessed in the explicit condition, including mean speed, $F(1.84,25.70)=5.11, p=.01, \eta^{2}=$ .27 ; peak speed, $F(2.67,37.65)=8.50, p<.001, \eta^{2}=.38$; trial-to-trial variability of mean speed, $F(2.55,35.76)=$ $3.07, p<.05, \eta^{2}=.18$; trial-to-trial variability of $R M S$ acc, $F(1.83,25.59)=3.64, p=.04, \eta^{2}=21$; and trial-to-trial variability of $R M S$ jerk, $F(2.90,40.56)=3.97, p=.02, \eta^{2}=$ .22. Pair-wise comparisons (Bonferroni) revealed generally lower mean and peak values and greater trial-to-trial variability during the high-complexity test than during the lowcomplexity test or during the first and second transfer tests, or both. However, trial-to-trial variability was also generally higher during the first transfer test, possibly because of the initial novelty of that block (see Table 1).

\section{Declarative Knowledge Protocol}

We contrasted the amount of explicit knowledge relevant to the mechanics of the movements in the two treatment conditions by using an independent-samples $t$ test. The test revealed a significant difference between the two groups, $t(33)=-2.68, p<.05, d=-.91$. Participants reported more knowledge in the explicit condition $(M=4.67, S D=2.10)$ than in the analogy condition $(M=3.18, S D=1.04)$.

\section{Discussion}

Poolton et al. (2006) showed that in a time-constrained environment, performance costs associated with processing both a difficult decision and an immediate motor response can be reduced if the participant acquires the motor task implicitly, that is, by analogy learning. During the decisionmaking test phase, adaptations made by participants because of the requirement to hit to targets left or right of center rather than centrally, as in the learning phase, may have confounded the findings. To overcome that problem, in this experiment we introduced a transfer test both before and after low- and high-complexity decision-making tests to ascertain whether adaptation continued throughout the test phase. In addition, we performed kinematic analysis of movements.
Participants learned to hit topspin forehand shots implicitly from our presentation of a single analogical instruction or explicitly from six step-by-step instructions that we provided. Consistent with previous findings in the explicitimplicit motor learning literature (Law, Masters, Bray, Eves, \& Bardswell, 2003; Liao \& Masters, 2001; Poolton et al., 2006), analogy learning resulted in less movement-related knowledge than did explicit learning, suggesting that a smaller amount of movement information was accessible to working memory for online control of movement.

Participants made fewer correct decisions in response to the high-complexity test than in response to the lowcomplexity test. The relative simplicity of low-complexity decisions meant that motor performance was not disrupted in either learning condition. No between-condition differences were evident when a complex decision was required. However participants' performance was disrupted in the explicit condition but not in the analogy condition. That finding replicates the results of Poolton et al. (2006) and implies that implicit motor learning via analogy facilitates the processing of multiple streams of information in a manner that is associated more with experts' performance than with novices' performance.

Analysis of the kinematic parameters showed that the movement characteristics remained constant in both conditions during the low-complexity decision task but that mean and peak movement speed decreased and trial-to-trial variability increased during the high-complexity decision task in the explicit condition but not in the analogy condition. For the explicit learners, the demands associated with the task may have caused stiffening of the movements. The reason for their movement stiffening is unclear, although people commonly become anxious if they perceive themselves to be unable to meet the demands of a task (e.g., Cherry, 1978; McGrath, 1970), and investigators have shown that anxiety increases motor stiffness (van Loon, Masters, Ring, \& McIntyre, 2001).

Masters and Liao (2003) proposed that analogies act as biomechanical metaphors that encapsulate (or chunk) many of the step-by-step rules of explicit performance. It is interesting that participants who learned by analogy in the current study produced movements with higher peak acceleration, mean acceleration, peak jerk, mean jerk, and trial-to-trial variability than did those who learned from explicit, step-by-step instructions. That finding may reflect a learning paradigm that quickly results in characteristics of expert performance. For example, Bootsma and van Wieringen (1990) reported that expert players compensate for later shot initiation by increasing the force (acceleration) applied in the shot. The greater acceleration (e.g., faster swing) evident in the analogy condition implies that analogy learners may have initiated movements later in an effort to give themselves more time to process the high-complexity decision before initiating the motor response. Direct comparison of the movement kinematics in each condition with those of expert performers would indicate how closely the movements reflected those of an expert. 
TABLE 1. Means and Standard Deviations of All Kinematic Variables During the Test Phase

\begin{tabular}{|c|c|c|c|c|c|c|c|c|}
\hline \multirow[b]{2}{*}{ Kinematic variable } & \multicolumn{2}{|c|}{ Transfer 1} & \multicolumn{2}{|c|}{ Low Complexity } & \multicolumn{2}{|c|}{ High Complexity } & \multicolumn{2}{|c|}{ Transfer 2} \\
\hline & $M$ & $S D$ & $M$ & $S D$ & $M$ & $S D$ & $M$ & $S D$ \\
\hline \multicolumn{9}{|c|}{ Analogy learning } \\
\hline Mean movement speed & 2.57 & 0.69 & 2.57 & 0.74 & 2.45 & 0.63 & 2.54 & 0.65 \\
\hline $\begin{array}{l}\text { Root mean square } \\
\text { acceleration }(R M S \mathrm{acc})\end{array}$ & 40.46 & 11.27 & 43.82 & 18.96 & 40.66 & 11.53 & 37.65 & 11.40 \\
\hline $\begin{array}{l}\text { Root mean square jerk } \\
(R M S \text { jerk })\end{array}$ & $1,274.16$ & 355.45 & $1,328.14$ & 450.69 & $1,316.21$ & 344.00 & $1,172.12$ & 346.41 \\
\hline Peak movement speed & 6.49 & 1.56 & 6.49 & 1.57 & 6.16 & 1.58 & 6.41 & 1.69 \\
\hline Peak $R M S$ acc & 91.64 & 24.88 & 107.82 & 77.81 & 92.22 & 24.90 & 88.44 & 28.04 \\
\hline Peak $R M S$ jerk & $3,851.64$ & $1,000.59$ & $3,904.82$ & $1,295.77$ & $3,794.70$ & 892.49 & $3,565.32$ & $1,161.18$ \\
\hline $\begin{array}{l}\text { Trial-to-trial variability } \\
\text { of mean movement speed }\end{array}$ & 0.32 & 0.11 & 0.31 & 0.11 & 0.35 & 0.13 & 0.41 & 0.17 \\
\hline $\begin{array}{l}\text { Trial-to-trial variability } \\
\text { of } R M S \text { acc }\end{array}$ & 6.57 & 2.43 & 7.19 & 6.30 & 7.26 & 2.99 & 6.79 & 2.89 \\
\hline $\begin{array}{l}\text { Trial-to-trial variability } \\
\text { of } R M S \text { jerk }\end{array}$ & 294.70 & 97.71 & 354.91 & 395.05 & 329.34 & 126.15 & 288.48 & 111.18 \\
\hline $\begin{array}{l}\text { Trial-to-trial variability } \\
\text { of peak movement speed }\end{array}$ & 0.66 & 0.26 & 0.72 & 0.48 & 0.72 & 0.31 & 0.71 & 0.27 \\
\hline $\begin{array}{l}\text { Trial-to-trial variability } \\
\text { of peak } R M S \text { acc }\end{array}$ & 20.72 & 7.76 & 25.53 & 35.01 & 20.81 & 9.48 & 19.58 & 8.30 \\
\hline $\begin{array}{l}\text { Trial-to-trial variability } \\
\text { of peak } R M S \text { jerk }\end{array}$ & $1,244.42$ & 441.75 & $1,539.76$ & $1,882.26$ & $1,229.70$ & 533.36 & $1,149.69$ & 512.91 \\
\hline \multicolumn{9}{|c|}{ Explicit learning } \\
\hline Mean movement speed & 2.41 & $0.56^{\mathrm{a}}$ & 2.22 & $0.48^{\mathrm{a}, \mathrm{b}}$ & 2.16 & $0.44^{\mathrm{b}}$ & 2.36 & $0.51^{\mathrm{a}}$ \\
\hline $\begin{array}{l}\text { Root mean square } \\
\text { acceleration }(R M S \mathrm{acc})\end{array}$ & 32.98 & $10.68^{\mathrm{a}}$ & 30.52 & $8.43^{\mathrm{a}}$ & 30.08 & $7.76^{\mathrm{a}}$ & 30.15 & $6.90^{\mathrm{a}}$ \\
\hline $\begin{array}{l}\text { Root mean square jerk } \\
(R M S \text { jerk })\end{array}$ & $1,016.36$ & $330.30^{\mathrm{a}}$ & 903.70 & $226.88^{\mathrm{a}}$ & 923.32 & $278.77^{\mathrm{a}}$ & 931.31 & $179.08^{\mathrm{a}}$ \\
\hline Peak movement speed & 6.21 & $1.04^{\mathrm{a}}$ & 5.99 & $1.08^{\mathrm{a}}$ & 5.51 & $0.79^{b}$ & 6.00 & $0.92^{\mathrm{a}}$ \\
\hline Peak $R M S$ acc & 74.43 & $19.86^{\mathrm{a}}$ & 70.67 & $19.50^{\mathrm{a}}$ & 68.61 & $13.85^{\mathrm{a}}$ & 70.90 & $15.05^{\mathrm{a}}$ \\
\hline Peak RMSjerk & $3,060.31$ & $770.36^{\mathrm{a}}$ & $2,859.05$ & $648.49^{a}$ & $2,799.31$ & $557.88^{\mathrm{a}}$ & $2,921.74$ & $555.79^{a}$ \\
\hline $\begin{array}{l}\text { Trial-to-trial variability } \\
\text { of mean movement speed }\end{array}$ & 0.36 & $0.14^{\mathrm{a}}$ & 0.28 & $0.09^{\mathrm{a}}$ & 0.33 & $0.12^{\mathrm{a}}$ & 0.26 & $0.09^{\mathrm{a}}$ \\
\hline $\begin{array}{l}\text { Trial-to-trial variability } \\
\text { of } R M S \text { acc }\end{array}$ & 5.64 & $2.83^{\mathrm{a}, \mathrm{b}}$ & 3.94 & $1.51^{\mathrm{a}}$ & 5.32 & $1.79^{\mathrm{b}}$ & 4.62 & $1.06^{\mathrm{a}, \mathrm{b}}$ \\
\hline $\begin{array}{l}\text { Trial-to-trial variability } \\
\text { of } R M S \text { jerk }\end{array}$ & 225.21 & $99.57^{\mathrm{a}, \mathrm{b}}$ & 166.46 & $62.70^{\mathrm{a}}$ & 238.77 & $101.55^{\mathrm{b}}$ & 212.12 & $70.56^{\mathrm{a}, \mathrm{b}}$ \\
\hline $\begin{array}{l}\text { Trial-to-trial variability } \\
\text { of peak movement speed }\end{array}$ & 0.57 & $0.36^{\mathrm{a}}$ & 0.47 & $0.14^{\mathrm{a}}$ & 0.79 & $0.64^{\mathrm{a}}$ & 0.53 & $0.20^{\mathrm{a}}$ \\
\hline $\begin{array}{l}\text { Trial-to-trial variability } \\
\text { of peak } R M S \text { acc }\end{array}$ & 15.53 & $6.58^{\mathrm{a}}$ & 12.47 & $3.94^{\mathrm{a}}$ & 18.52 & $11.05^{\mathrm{a}}$ & 15.99 & $5.40^{\mathrm{a}}$ \\
\hline $\begin{array}{l}\text { Trial-to-trial variability } \\
\text { of peak } R M S \text { jerk }\end{array}$ & 887.42 & $344.58^{\mathrm{a}}$ & 818.01 & $371.35^{\mathrm{a}}$ & 1108.23 & $539.53^{\mathrm{a}}$ & 872.47 & $308.72^{a}$ \\
\hline
\end{tabular}

Note. There were no significant differences between blocks for any measure at $p<.05$. Low- and high-complexity decision tests were interspersed between two transfer tests (T1 and T2). Distances are expressed in meters; times are expressed in seconds. Shared superscripts (a,b) denote no significant differences at $p<.05$.

Although our findings support the working-memory explanation (Masters \& Maxwell, 2004) for why implicit motor learning allows more efficient decision making and motor performance in time-constrained environments, an alternative explanation is that the modular architecture of working memory (Baddeley, 2003) allows parallel per- formance of the tasks without taxing the same modules. Whereas cognitively demanding decisions (Jameson et al., 2004) and manipulation of explicit information (MacMahon \& Masters, 2002) occur in the central executive module of working memory, analogical instruction does not. Despite the verbal manner in which the analogy is communicated, 
Liao and Masters (2001) argued that the analogy is likely to be processed as an image in the visuospatial sketchpad module of working memory, where it can be used to support movement control. Consequently, the neuromotor system may process the two tasks in different modules within working memory without overreaching the capacity of the working-memory area to process them simultaneously.

Another possibility is that analogy learners more easily switched between the two tasks than explicit learners did. Poolton et al. (2006) dismissed that possibility on the ground that the time window for task execution was unlikely to be sufficient to accommodate task-switching behavior. The temporal constraints of the task in this study also suggest that task switching was not feasible. Researchers have found that durations between shot initiation and bat-ball contact of $370 \mathrm{~ms}$ increase to approximately $399 \mathrm{~ms}$ when participants have to adapt the parameters of the movement to hit the ball to either the left or the right side of the table (e.g., Roth, 1989). We approximated that participants had a 450-ms time window between ball release and ball strike in which they could execute the movement. Given that simple reaction times approximate $190 \mathrm{~ms}$ and escalate as the number of stimulus-response choices increase (Hick, 1952; Welford, 1980), an overlap between the two components of the task was probable, and task switching is unlikely to have been an effective strategy.

Our findings suggest that although analogical instructions are conveyed explicitly, they are cognitively efficient (as defined by Moors \& De Houwer, 2006), in that they demand few processing resources (Law et al., 2003; Liao \& Masters, 2001; Masters \& Liao, 2003; Poolton et al., 2006). As a consequence, learning by analogy appears to install in the motor behavior of novices (implicit) characteristics that normally are not evident in perception-action behavior until the performer is much farther along the road to expertise.

\section{ACKNOWLEDGMENT}

A grant from the Germany/Hong Kong Joint Research Scheme, offered by the Research Grants Council of Hong Kong and the Deutscher Akademischer Austauschdienst, supported the research described in this article.

\section{Biographical Notes}

R. S. W. Masters is assistant director for research at the Institute of Human Performance, The University of Hong Kong, China. He is interested in understanding the knowledge bases that support skill acquisition and skilled performance, particularly in relation to the phenomenon of implicit motor learning. He is presently an associate editor for Psychology of Sport \& Exercise.

J. M. Poolton recently completed his doctoral degree at the Institute of Human Performance, The University of Hong Kong. $\mathrm{He}$ is interested in implicit motor learning, with emphasis on the applications to skill in sport.

J. P. Maxwell is a research assistant professor at the Institute of Human Performance, The University of Hong Kong. His research interests involve the cognitive mechanisms underlying skill acquisition and performance and the role of errors in learning.

M. Raab is a professor of movement science and sport psychology at the Institute for Movement Science and Sport, University of Flensburg, Germany. His main interests are motor learning, judgment, and decision making in sports.

\section{REFERENCES}

Anderson, J. R. (1983). The architecture of cognition. Cambridge, MA: Harvard University Press.

Baddeley, A. D. (2003). Working memory: Looking back and looking forward. Nature Reviews Neuroscience, 4, 829-839.

Bootsma, R. J., \& van Wieringen, P. C. W. (1990). Timing an attacking forehand drive in table tennis. Journal of Experimental Psychology: Human Perception and Performance, 16, $21-29$.

Cherry, N. (1978). Stress, anxiety and work: A longitudinal study. Journal of Occupational Psychology, 51, 259-270.

Hardy, L., Mullen, R., \& Jones, G. (1996). Knowledge and conscious control of motor actions under stress. British Journal of Psychology, 87, 621-636.

Hazeltine, E., Ruthruff, E., \& Remington, R. W. (2006). The role of input and output modality pairings in dual-task performance: Evidence for content-dependent central interference. Cognitive Psychology, 52, 291-345.

Hick, W. E. (1952). On the rate of gain of information. Quarterly Journal of Experimental Psychology, 4, 11-26.

Jameson, T. L., Hinson, J. M., \& Whitney, P. (2004). Components of working memory and somatic markers in decision making. Psychonomic Bulletin and Review, 11, 515-520.

Law, J., Masters, R. S. W., Bray, S. R., Eves, F. F., \& Bardswell, I. (2003). Motor performance as a function of audience affability and metaknowledge. Journal of Sport and Exercise Psychology, 25, 484-500.

Lewicki, P., Hill, T., \& Czyewska, M. (1992). Nonconscious acquisition of information. American Psychologist, 47, 796-801.

Liao, C., \& Masters, R. S. W. (2001). Analogy learning: A means to implicit motor learning. Journal of Sports Sciences, 19, 307-319.

MacMahon, K. M. A., \& Masters, R. S. W. (2002). The effects of secondary tasks on implicit motor skill performance. International Journal of Sport Psychology, 33, 307-324.

Masters, R. S. W. (1992). Knowledge, knerves and know-how: The role of explicit versus implicit knowledge in the breakdown of a complex motor skill under pressure. British Journal of Psychology, 83, 343-358.

Masters, R. S. W., \& Liao, C. (2003). Chunking as a characteristic of implicit motor learning. In R. Stelter (Ed.), New approaches to exercise and sport psychology: Theories, methods and applications (p. 137). Copenhagen, Denmark: University of Copenhagen.

Masters, R. S. W., \& Maxwell, J. P. (2004). Implicit motor learning, reinvestment and movement disruption: What you don't know won't hurt you. In A. M. Williams \& N. J. Hodges (Eds.), Skill acquisition in sport (pp. 207-228). New York: Routledge.

Maxwell, J. P., Masters, R. S. W., \& Eves, F. F. (2003). The role of working memory in motor learning and performance. Consciousness and Cognition, 12, 376-402.

Maxwell, J. P., Masters, R. S. W., Kerr, E., \& Weedon, E. (2001). The implicit benefit of learning without errors. Quarterly Journal of Experimental Psychology A, 54, 1049-1068.

McGrath, J. E. (1970). Social and psychological factors in stress. New York: Holt, Rinehart \& Winston.

Moors, A., \& De Houwer, J. (2006). Automaticity: A theoretical and conceptual analysis. Psychological Bulletin, 132, 297-326.

Poolton, J. M., Masters, R. S. W., \& Maxwell, J. P. (2006). The influence of analogy learning on decision-making in table tennis: Evidence from behavioural data. Psychology of Sport and Exercise, 7, 677-688.

Poolton, J. M., Masters, R. S. W., \& Maxwell, J. P. (2007). Pass- 
ing thoughts on the evolutionary stability of implicit motor behaviour: Performance retention under physiological fatigue. Consciousness and Cognition, 16, 456-468.

Raab, M. (2003). Decision making in sports: Influence of complexity of implicit and explicit learning. International Journal of Sport and Exercise Psychology, 1, 310-337.

Roth, K. (1989). Taktik im sportspiel [Tactics in games]. Schorndorf, Germany: Hoffman.

Sève, C., Saury, J., Theureau, J., \& Durand, M. (2002). Activity organization and knowledge construction during competitive interaction in table tennis. Cognitive Systems Research, 3, 501-522.

Shiffrin, R. M., \& Schneider, W. (1977). Controlled and automatic human information processing: II. Perceptual learning, automatic attending, and a general theory. Psychological Review, 84, 127-190.

Sneyd, S. (1994). Basic coaching manual. East Sussex, England: English Table Tennis Association.

The Sport Council. (1995). Teaching students to play games 11-16: Table tennis. London, England: Author.

van Loon, E. M., Masters, R. S. W., Ring, C., \& McIntyre, D. B. (2001). Changes in limb stiffness under conditions of mental stress. Journal of Motor Behavior, 33, 153-164.

Welford, A. T. (1980). Choice reaction time: Basic concepts. In A. T. Welford (Ed.), Reaction times (pp. 73-128). New York: Academic Press.

Willingham, D. B. (1998). A neuropsychological theory of motor skill learning. Psychological Review, 105, 558-584.

\section{APPENDIX}

Instructions to Participants in the Explicit Learning Condition and the Analogy Learning Condition

\section{Explicit learninga}

1. Keep your feet a little wider than shoulder width apart.

2. Position your feet behind the table with the right foot farthest from the table.

3. Move the bat backward and down.

4. Move your body weight to the front leg.

5. Move your playing arm forward and upward.

6 . Keep the bat face at a vertical angle.

\section{Analogy learning}

Move the bat as if it is traveling up the side of a mountain.

${ }^{a}$ We took the instructions for the explicit learning condition from S. Sneyd (1994) and The Sport Council (1995). A Cantonese translation of the instructions is available from the authors.

Submitted June 30, 2006

Revised December 1, 2006

Second revision February 7, 2007

Third revision February 21, 2007 
Reproduced with permission of the copyright owner. Further reproduction prohibited without permission. 